\section{P3.45 IDENTIFYING HEALTH CARE SETTINGS FOR PREP DELIVERY TO MSM AT HIGH RISK FOR HIV ACQUISITION}

${ }^{1} \mathrm{CM}$ Schumacher, ${ }^{2} \mathrm{~S}$ Bacchus, ${ }^{3} \mathrm{~A}$ Chandran, ${ }^{2} \mathrm{Y}$ Kingon, ${ }^{1,3} \mathrm{E}$ Fields, ${ }^{2} \mathrm{~K}$ Bandemuse, ${ }^{2} \mathrm{R}$ Muuva, ${ }^{2,3} \mathrm{P}$ Chaulk, ${ }^{1,3} \mathrm{JM}$ Jennings. 'Johns Hopkins University School of Medicine, Baltimore, Maryland, USA; ${ }^{2}$ Baltimore City Health Department, Baltimore, Maryland, USA; ${ }^{3}$ Johns Hopkins University Bloomberg School of Public Health, USA

\section{$10.1136 /$ sextrans-2017-053264.282}

Introduction Syphilis rates among MSM are increasing sharply in urban areas across the U.S. MSM with syphilis are at high risk for acquiring HIV, and may be an important subgroup to increase awareness and delivery of pre-exposure prophylaxis (PrEP). Key, however, is identifying access points to this population. Our objective was to determine health care settings where syphilis positive and HIV negative (vs. HIV positive) MSM were diagnosed to prioritise and tailor to settings for PrEP delivery. Setting: A mid-Atlantic U.S. city which has seen a $102 \%$ increase in early (primary, secondary and early latent) syphilis among MSM from 2009-2015.

Methods We analysed routinely collected public health surveillance data on MSM diagnosed with early syphilis reported to a city health department between 2009-2015. We compared diagnosing provider information by HIV status overall and in 2015 using Chi-squared tests.

Results Of the 1,495 MSM diagnosed with early syphilis between 2009-2015, the majority was aged $\geq 25$ years (73\%), African American (86\%) and HIV co-infected (67\%). Overall, $52 \%$ were diagnosed in private health care settings, and $25 \%$ were diagnosed in publically funded sexually transmitted infection (STI) clinics. Early syphilis positive/HIV negative MSM were more likely than HIV positive MSM to receive a syphilis diagnosis in STI clinics (38\% vs. $19 \%$ $\mathrm{p}=<0.0001$ ) and Emergency Departments (EDs) or Urgent Care Centres (UCC) $(12 \%$ vs. $8 \% \mathrm{p}<0.0001)$ and less likely to be diagnosed by private providers $(33 \%$ vs. $61 \%$, $\mathrm{p}<0.0001)$. Among the 268 MSM diagnosed with early syphilis in 2015, HIV negative MSM $(n=44)$ were as likely as HIV positive MSM $(n=224)$ to receive a syphilis diagnosis in STI clinics $(27 \%$ vs. $16 \%, \mathrm{p}=0.06)$, more likely to be diagnosed in EDs/UCCs $(20 \%$ vs. $10 \%, \mathrm{p}=0.03)$ and less likely to be diagnosed by private providers $(36 \%$ vs. $66 \%, \mathrm{p}<0.0001)$.

Conclusions EDs/UCCs and are important access points for MSM at high risk for HIV but sites may change over time. Efforts by the city health department to increase PrEP delivery at these sites are being initiated.

\section{P3.46 SEROPREVALENCE AND INCIDENCE OF CHLAMYDIA TRACHOMATIS IGG AND IGA IN MEN WHO HAVE SEX WITH MEN}

${ }^{1}$ HOEBE CJPA, ${ }^{2}$ IHM VAN LOO, ${ }^{2}$ TM GOOSSENS, ${ }^{1} \mathrm{G}$ VAN LIERE, 'DUKERSMUIJRERS NHTM. 1 PHS South Limburg, Geleen, The Netherlands; ${ }^{2}$ MUMC, MAASTRICHT, The Netherlands. Liere (PHS South Limburg), NHTM Dukers-Muijrers (PHS South Limburg)

\subsection{6/sextrans-2017-053264.283}

Introduction Although routine diagnostic methods for detection of Chlamydia trachomatis (CT) are based on Nucleic Acid Amplification Tests (NAAT) the detection of antibodies can also be used as an additional tool, especially for surveillance. People with a CT infection develop serum IgG and $\operatorname{IgA}$, which are a marker for past infection and in women are correlated with infertility. Although seroprevalence of CT has been well studied in women, little is known about the seroprevalence of CT in men, especially in the high risk group men who have sex with men (MSM). The aim of this study is to assess the seroprevalence of CT in MSM and the development of seroconversion over time.

Methods A seroprevalence study was conducted in 291 MSM visiting the STI clinic of the Public Health Service South Limburg, the Netherlands, at least twice between January 2011 and December 2013. Sera from the last consultation (T2) were tested for the presence of $\operatorname{IgG}$ and $\operatorname{IgA}$ (Medac, Germany). Individuals with positive serology at T2 were additionally tested one year before (T1) to determine seroconversion. Prevalence data were calculated from the number of $\operatorname{IgG}$ and IgA positive sera at $\mathrm{T} 2$ and incidence data were calculated from the seroconversion rates between $\mathrm{T} 1$ and $\mathrm{T} 2$.

Results Thirty-one percent $(n=91 / 291)$ of MSM was NAAT CT positive in the study period.

In 98\% (286/291) MSM sera were available for testing. In total, $32 \%$ of MSM (91/286) were IgG positive and $17 \%$ were IgA positive (48/286), of which 44 were positive for both. The overall prevalence was $33 \%$ based on the presence of IgG and/or IgA antibodies $(\mathrm{n}=95)$. Seroconversion rate between $\mathrm{T} 1$ and $\mathrm{T} 2$ showed that 3,8\% $(\mathrm{n}=11)$ seroconverted for $\operatorname{IgG}$ and $4,5 \%(n=13)$ for $\operatorname{IgA}$, of which $1.7 \% \quad(n=5)$ seroconverted for both. The overall incidence rate was $6,6 \%$ $(n=19)$ based on seroconversion of $\operatorname{IgG}$ and/or IgA.

Conclusion This study showed that one third of MSM visiting an STI clinic were seropositive for CT. The incidence rate was about $6 \%$. Association of CT seropositivity with sexual behaviour determinants and actual CT positivity will be further studied.

\section{P3.47 AWARNESS OF HIVIAIDS AMONG RURAL POPULATION OF GURGAON, INDIA}

Clement Joy Kingsly. Nestle India Limited, Gurgaon, India

\subsection{6/sextrans-2017-053264.284}

Introduction Despite remarkable world-wide progress in the field of diagnostic, curative and preventive medicine, still there are large populations of people living in isolation in natural and unpolluted surroundings far away from civilisation, maintaining their traditional values, customs, beliefs and myths. They are commonly known as tribes and are considered to be the indigenous people of the land. This study aimed to assess HIV-related knowledge, attitudes and practices among rural population of Gurgaon, India.

Methods A cross-sectional study on rural general population aged between 20 and 30 years old was undertaken to evaluate their KAPs. We selected 400 eligible samples through systematic random sampling from different villages.

Results The majority of the population was unaware of HIV (54\%). Of the population who were aware 39\% knew that it can be transmitted by sexual intercourse and $28 \%$ from mother to child. Misconceptions about transmission of HIV were observed among $33 \%$ to $43 \%$ of respondents. More $65 \%$ mentioned village health workers as major sources of information on HIV/AIDS.

Conclusions Despite adequate knowledge about HIV/AIDS, misconceptions about routes of transmission were found. Negative attitudes to HIV/AIDS and risky practices were also 
present. Educational programmes with specific interventions are recommended to increase KAPs and to prevent new HIV infections among this population

\section{P3.48 CHARACTERIZACION OF THE GESTATIONAL OUTCOME OF INFANTS BORN TO HIV POSITIVE MOTHERS IN ANTIRETROVIRAL TREATMENT DURING PREGNANCY}

${ }^{1} \mathrm{JC} O \mathrm{C}$ Crispim, ${ }^{2} \mathrm{KR}$ Torres, ${ }^{3} \mathrm{GM}$ Palomino, ${ }^{4} \mathrm{PE}$ A Siqueira, ${ }^{1} \mathrm{MN}$ Monteiro, ${ }^{5} \mathrm{MT}$ A Lima, ${ }^{1}$ DA Pascoal. 'Maternity School Januário Cicco, Universidade Federal do Rio Grande do Norte, Natal - RN, Brazil; ${ }^{2}$ Medicine Department, Universidade Federal do Rio Grande do Norte, Natal - RN, Brazil; ${ }^{3}$ Postgraduate Program in Pharmaceutical Science, Universidade Federal do Rio Grande do Norte, Natal - Rn, Brazil; ${ }^{4}$ Pharmacy Department, Universidade Federal do Rio Grande do Norte, Natal - RN, Brazil; ${ }^{5}$ Hospital of Paediatrics Teacher Heriberto Ferreira Bezerra, Universidade Federal do Rio Grande do Norte, Natal - RN, Brazil

10.1136/sextrans-2017-053264.285

Introduction Antiretroviral regimens used for the prevention of mother-to-child transmission of the human immunodeficiency virus (HIV) have shown benefits. However, combination antiretroviral therapy (ART) for the mother and infant have shown higher rates of adverse pregnancy outcomes. The objective of this research is to characterise the gestational outcome of infants born to HIV-positive mothers receiving highly active antiretroviral therapy (HAART) in a tertiary maternity unit through the evaluation of the clinical, anthropometric and epidemiological profile of the babies at birth.

Methods A cross-sectional study was carried out on HIV positive pregnant women at the Januário Cicco Maternity School (MEJC), whose childbirths occurred in the period from January 2010 to December 2014, being included 82 mothers and 84 babies. The research will continue in the year 2017.

Results The group of mothers selected had a mean age of 29.5 years and had their children with a mean gestational age of 36.7 weeks. Of these, $3.6 \%$ received a triple therapeutic regimen with combination of nucleoside reverse transcriptase inhibitor (NRTI) +2 protease inhibitors (PI), 4.9\% received regimen with 2 NRTI +1 Non-nucleoside reverse transcriptase inhibitor and $91.5 \%$ received quadruple regimen with 2 NRTI +2 PI. According to the information collected in the present study, only $17.8 \%$ of the babies were born prematurely. As for anthropometry, 22.6\% were born with low weight; $28.6 \%$ had cephalic perimeter and $15.5 \%$ length below the lower limits considered normal; and $98.8 \%$ of the babies reached.

Conclusion This type of characterisation is fundamental in a scenario where changes in the neonatal characteristics observed in the study generate a cost for maternal and child health, involving economics and management aimed at a specific follow-up of HIV positive mothers and their children exposed to the virus and to HAART during gestation. For this reason, it is necessary the specialised and multiprofessional clinical follow-up for the binomial in question.

\section{P3.49 CONTRIBUTION TO THE STUDY OF EPIDEMIOLOGICAL SURVEILLANCE OF CONGENITAL SYPHILIS IN A HOSPITAL OF THE PUBLIC HEALTH SYSTEM IN RJ, BRAZIL, JANUARY 2013 TO JANUARY 2017}

Cunha Aa, C Galvão, WC Borges, P Godefroy, Teixeira Sam, M Glinardello, E Gerde. Hospital Maternidade Therezinha de Jesus, Rio de Janeiero - RJ, Brazil

10.1136/sextrans-2017-053264.286
Introduction Syphilis consists of an STD (sexually transmitted disease) of bacterial aetiology, featuring a serious public health problem. The objective was to describe the prevalence of congenital syphilis in a hospital of the Public Health System Rio de Janeiro, Brazil.

Methods Design. A descriptive and observational research of quantitative and retrospective nature. Data collection. We collected data from the medical records. Statistical analysis. The data were processed by simple frequency measures (absolute and relative).

Results We analysed 175 notified CS (congenital syphilis) cases in 6274 deliveries (prevalence of 2,7\%). Some women at the time of delivery had their data collected, were affected by syphilis during pregnancy and prenatal care. About of $80.0 \%$ of women with syphilis performed prenatal care. There were carried out positive 276 tests (VDRL) in the Hospital Estadual da Mãe by the women in the study, 104 (59.42\%) in prenatal care and $172(98.28 \%)$ at time of delivery, when the test was repeated for all the participants. The distribution of the number of sexual partners of syphilitic pregnant women that made treatment during prenatal care of pregnant women was very impaired because of very high missing values.

Conclusions At the end of this study we found that even though the Ministry of Health has established a program together with the World Health Organisation, the goal of reducing the number of cases of congenital syphilis has not yet been reached. We have problems with the prenatal care, lack of effective screening program and monitoring of the pregnant woman. The findings of CS in the State Hospital of Mother separated for investigation were considered high in relation to the proposal drawn up by the Ministry of Health $(1 / 1,000)$.

\section{P3.50 SPECTRUM OF MALIGNANCIES AMONG PEOPLE LIVING WITH HIV (PLHIV) IN SOUTHERN INDIA}

D Madi, A Aliasgar, J Ramapuram. Department of Medicine, KMC Mangalore (Manipal University), India

\subsection{6/sextrans-2017-053264.287}

Introduction Non-Hodgkin's Lymphoma (NHL), Kaposi’s sarcoma and invasive carcinoma of cervix are AIDS defining cancers (ADCs) seen in people living with HIV (PLHIV). After the introduction of combination antiretroviral therapy (cART) the spectrum of malignancies has changed and the incidence of Non AIDS defining Cancers (NADCs) are increasing as PLHIV live longer.This study describes the pattern of malignancies among PLHIV attending a tertiary care HIV facility.

Methodology This descriptive cross sectional study was carried out at Kasturba Medical College (KMC), Mangalore which is a 500 bedded tertiary care referral institution. The study was conducted among PLHIV diagnosed with malignancy between January 2005 and May 2016. Data of 33 PLHIV was collected by using a semi-structured proforma after obtaining permission from the institutional ethics committee. Data was analysed by using SPSS version 11.5 statistical software.

Results The mean age of our study group was $46.19 \pm 7.82$ years. Majority of them $18(54.5 \%)$ were males. In the current study $19(57.6 \%)$ had Non AIDS defining cancers and 14 (42.4\%) had AIDS defining cancers. Non-Hodgkin's lymphoma 9 (27.2\%), carcinoma breast 5 (15.2\%), invasive carcinoma of cervix $5(15.2 \%)$ and carcinoma of head and neck 4 